\title{
CMSE2R: Clustered-based Multipath Shortest- distance Energy Efficient Routing Protocol for Underwater Wireless Sensor Network
}

\author{
Mukhtiar Ahmed', M. Ali Soomro'2, Sajida Parveen², Javed Akhtar' and Nadeem Naeem ${ }^{3}$ \\ 'Department of Information Technology, Quaid-e-Awam, University of Engineering, Science, and Technology, \\ Nawabshah, Sindh, Pakistan; mukhtiar.a@gmail.com, jawedunar@gmail.com \\ 2Department of Computer Systems Engineering, Quaid-e-Awam, University of Engineering, Science, and \\ Technology, Nawabshah, Sindh, Pakistan; kalakot2@gmail.com, sajidaparveen@quest.edu.pk \\ ${ }^{3}$ Department of Electronics Engineering, Quaid-e-Awam, University of Engineering, Science, and Technology, \\ Nawabshah, Sindh, Pakistan; nadeembhatti@quest.edu.pk
}

\begin{abstract}
Objectives: From the existing literature review we found that the link quality between nodes and to prolong the battery power of the nodes still needs improvement. This research article controls the node mobility in underwater environment through maintaining link between nodes and prolongs the battery power of the nodes through Cluster-based Multipath Shortest-distance Energy Efficient Routing (CMSE2R). Method/Analysis: In this research article, we proposed the Clustered-based Multipath Shortest-distance Energy Efficient Routing (CMSE2R) protocol for Underwater Wireless Sensor Network. CMSE2R is based on four phases to improve the link quality between nodes and reduces the average energy consumption. These phases are: Network setup phase, cluster formation phase, multipath development in relevant clusters, and data forwarding phase. Findings: The results of CMSE2R are compared with CBE2R and EMGGR and it is observed that CMSE2R is a better performer than CBE2R and EMGGR.This protocol improves the link quality through use of fixed courier nodes and formation of multipath in relevant cluster formation mechanism. This routing protocol uses the Cumulative Distance Calculation Mechanism (CDCM) to reduce the average energy consumption. Applications/Improvements: The proposed routing protocol extracts the valuable information from the seabed to sea surface.
\end{abstract}

Keywords: Calculation, CDCM, Layers, Shortest-Distance, Snods

\section{Introduction}

Underwater wireless routing is the main focus of the research community due to its multipurpose applications like: tactical surveillance, assisted navigation, disaster prevention, offshore exploration, pollution monitoring, oceanographic data collection like oil/gas, gold, minerals, silver, diamond, under coal mines etc ${ }^{1-6}$. In underwater environment, the designing of routing protocol faces many more challenges like: 3D sensing range of nodes, communication link between nodes, underwater noise, propagation delay, channel limitation, multipath fading, underwater biological conditions, water pressure, water current, and limited battery power etc $\frac{8-12}{}$. Majority of the routing protocols have followed the approach of the terrestrial based network mechanisms, which are failure to proper work in underwater environment because in underwater environment the deployment of nodes is very complicated due to water pressure and node mobility $\underline{10-13}$. It is also observed that underwater environment RF and optical signaling cannot work properly, because RF signaling have limited frequency and it needs the large antenna which we cannot deploy in underwater environment, on other hand the optical signaling can work with high pre-

${ }^{*}$ Author for correspondence 
cession with line of sight, which is also not possible in underwater harsh environment $\frac{13-15}{}$. In underwater environment the two main issues still needs the improvement, one is the link quality and other is to prolong the battery power of the nodes. Clustered-based Multipath Shortestdistance Energy Efficient Routing (CMSE2R) protocol for underwater wireless sensor network prolongs the battery power of the nodes through efficient use of powerful static courier nodes, efficient cluster formation mechanism with multipath route selection mechanism. CMSE2R develops the link quality between nodes through shortest distance route selection mechanism.

In related work, we have considered the relevant literature with its limitations as described below:

\subsection{An energy-efficient multi-level adaptive routing algorithm for underwater wireless sensor network (ACUN)}

In ${ }^{16}$ the energy-efficient Adaptive Clustering Underwater Networking (ACUN) routing algorithm is proposed to improve the energy efficiency. ACUN uses the multi-level hierarchical network based structure for distance between cluster-head and sink node to prolong the battery power of the nodes. ACUN considers the residual energy of the cluster-head to determine the size of the competition radius. ACUN looks the larger residual energy during selection of the cluster-head node to prolong the battery power of the node. ACUN balance the energy level of the nodes through single or multi-hop route selection mechanism. It is observed from the ACUN that the cluster-head selection through radius with layered formation mechanism is the complex due to distance measurement, because when network becomes sparse then this kind of mechanism is unable to maintain the overall network performance. It is also observed that when network becomes sparse the cluster-head node will drop the packets continuously and will die earlier.

\subsection{Energy-Aware and Void-Avoidable Routing Protocol (EAVARP)}

$\mathrm{In}^{17}$ EAVARP is proposed, in this routing protocol the two phases are mentioned to perform the operation, phase one is layering phase and phase two is data collection phase. Layering phase forms the different number of concentric shells around the sink node. Sink node itself performs the hierarchical task to validate the real-time topology. The opportunistic directional forwarding strategy (ODFS) mechanism is used to forward the data packets to the water surface. EAVARP is only based on ODFS to prolong the battery power of the nodes within shells through avoidance of cyclic transmission. It is observed from the EAVARP that the complex mechanism for shell formation through ODFS is mentioned, which only works in the limited area, even authors have not mentioned the performance parameters for dense or sparse area networks.

\subsection{Clustered Based Energy Efficient Routing (CBE2R)}

$\mathrm{In}^{-3} \mathrm{CBE} 2 \mathrm{R}$ is proposed, this routing protocol is the water depth is controlled through formation of layers from water surface to seabed, the use of courier nodes prolongs the battery power of the nodes, for route development mechanism the cluster formation mechanism is used from which the bottom layer courier node plays the role of cluster-head nodes and cluster-head node will transfer the message to the ordinary nodes to form the cluster member nodes from bottom layer cluster-head node to source nodes which are deployed at the seabed level. The low weighted distance calculation mechanism is used to develop the route between low layer cluster-head node (courier node) to seabed source node for developing the stable route for packets forwarding, when cluster-head node will collect the packets then this node will forward the packets to the sink nodes which are deployed at water surface by using the maximum power levels through other layered courier nodes. It is observed that CBE2R cannot perform well when network becomes sparse.

\subsection{Energy-efficient Multipath Grid-based Geographic Routing (EMGGR)}

$\mathrm{In}^{\underline{18}}$ EMGGR is proposed. This routing protocols form the $3 \mathrm{D}$ grids in underwater environment to control the $3 \mathrm{D}$ environment of the underwater. In grids the multipath route development mechanism is adapted to forward packets from source to sink node. In grid cells the $x y z$ addressing mechanism is used and every node is comprises the $x y z$ addressing mechanism and are well aware about its location. The data is forwarded through virtual cell gateway from source to sink. It is observed from EMGGR that due to the water pressure the 3D grid formation mechanism cannot perform well. 


\subsection{Energy-efficient Distance Routing Protocol (DRP)}

In $\frac{19}{}$ DRP is proposed. In DRP the Hello packet is forwarded from sink node to the neighbor nodes to locate the maximum residual energy of the nodes to develop the route between source to sink node for packets forwarding. DRP has power to detect the collision and controls the collisions probability between nodes. The multipath route development mechanism has been adapted in DRP for packets forwarding. It is observed that DRP cannot control its distance due to node mobility and cannot show its well performance.

\subsection{Reliable Energy Efficient Protocol (REEP)}

$\mathrm{In}^{20} \mathrm{REEP}$ is proposed. This routing protocol uses the To $\mathrm{A}$ mechanism for calculating the distance between source and sink node. The network development and data forwarding phases focuses the operation of the REEP. In REEP the forwarder node selection is based on location information and residual energy. For data forwarding the three formats are uses, one is hello packet format, second is reply format and third is data packet format. REEP is only based on the use of vertical modem, whereas, if network become sparse then forwarder node will drop the packets and will die earlier.

From the aforementioned routing protocols, it is clear that there is need to design a novel routing protocol which can control the link between nodes and prolong the battery power of the nodes.

\section{Clustered-based Multipath Shortest-distance Energy Efficient Routing (CME2R)}

CME2R operation is based on four phases. Phase one is network setup, phase two is formation of cluster, phase three is formation of multipath in relevant cluster, and phase four is data forwarding from source to sink.

\subsection{Network Setup Phase}

In this phase, we have divided the water depth in upper and lower depth. The upper depth is divided into two layers and on each layer, the static powerful static courier nodes $(\mathrm{CNs})$ are deployed and are connected between each other through acoustic channel. The use of pow- erful CNs prolongs the battery power of the ordinary/ forwarder nodes (FNs). On water surface the sink nodes (Snods) are deployed. The Snodsare connected between each other through radio frequency (RF) signaling.Snods are also connected with the onshore data center through RF signaling. In lower depth the Source Nodes (SNs) are deployed at the seabed level. The FNs are deployed between layer-2 CNs from upper depth. The network setup is shown in Figure 1.

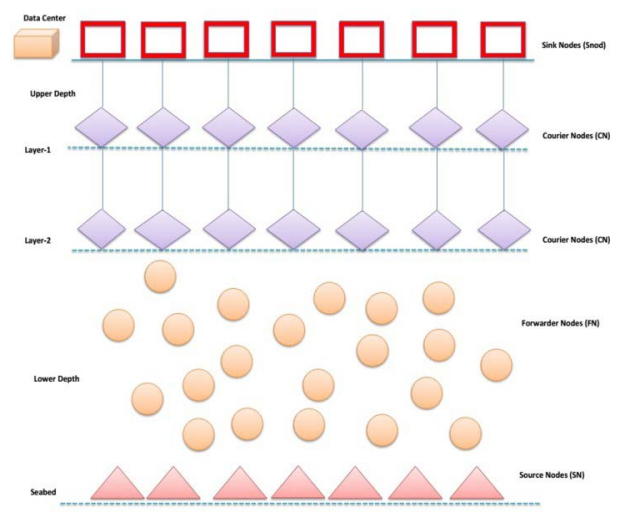

Figure 1. Network setup phase.

\subsection{Cluster Formation Phase}

In cluster formation phase the layer- 2 every $\mathrm{CN}$ will forward the hello message to the FNs and will become cluster-head node, when FNs will receive the hello message then FNs will become the cluster-member nodes to form the cluster around relevant $\mathrm{CN}$. The formation of cluster is shown in Figure 2.

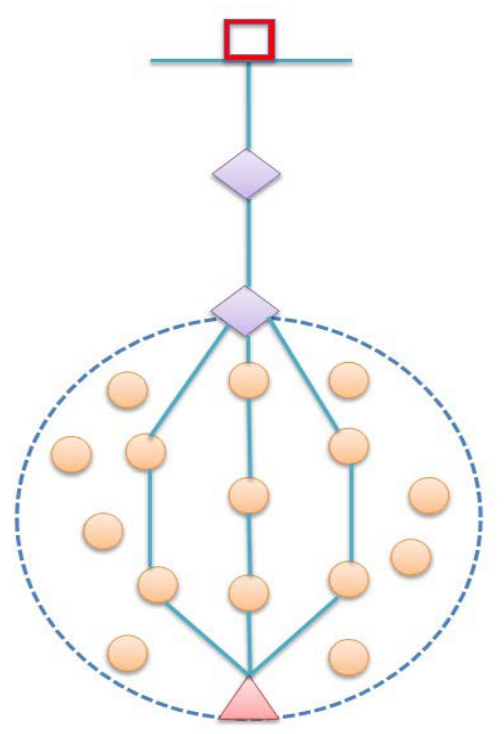

Figure 2. Cluster formation phase. 


\subsection{Formation of Multipath in Relevant}

\section{Cluster}

The formation of multipath in relevant cluster phase focuses the formation of multipath route development phase from SN to CN (Cluster-head) through RREQ and $R R E P$ packet. The RREQ and RREP packet comprises with the Cumulative Distance Calculation Mechanism (CDCM) to calculate the shortest distance between layer-2 CN (Cluster-head). The multipath route is developed on RREQ from SN towards CN with calculation of CDCM. This whole phenomenon is shown in Figure 3.

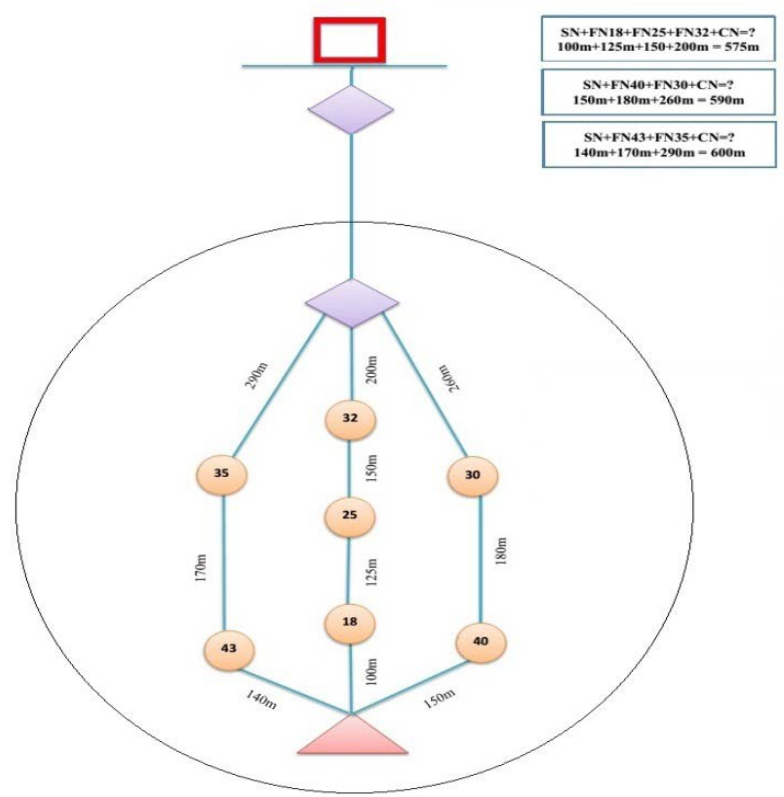

Figure 3. Multipath route development in relevant cluster.

The CDCM of the nodes from SN to CN (Clusterhead) is described as:

(i) $\mathrm{SN}+\mathrm{FN} 18+\mathrm{FN} 25+\mathrm{FN} 32+\mathrm{CN}=$ ?

$100 m+125 m+150 m+200 m=575 m$

(ii) $\mathrm{SN}+\mathrm{FN} 40+\mathrm{FN} 30+\mathrm{CN}=$ ?

$150 \mathrm{~m}+180 \mathrm{~m}+260 \mathrm{~m}=590 \mathrm{~m}$

(iii) $\mathrm{SN}+\mathrm{FN} 43+\mathrm{FN} 35+\mathrm{CN}=$ ?

$140 m+170 m+290 m=600 m$

\subsection{Data Forwarding Phase From Source to Sink Nodes}

After the calculation of CDCM, the shortest distance between $\mathrm{SN}$ to $\mathrm{CN}$ (Cluster-head) which is mentioned in Equation (1), that is $575 \mathrm{~m}$ from SN to FN18 to FN25 to FN32 to CN nodes. Through RREP from CN to SN along with shortest CDCM which tells the SN that forward the packets from this route. The packets forwarding mechanism is shown in Figure 4.

When layer-2 $\mathrm{CN}$ will receive the packets then this node will forward the packets to the layer- $1 \mathrm{CN}$ through utilization of maximum power level and the packets will be received by surface Snod. This process is used for all SNs to CNs to Snods, because the network setup phase follows the multi-sink architecture which maximizes the overall performance of the entire network.

\section{Performance Analysis of CMSE2R}

In performance analysis, we have used the NS2.30 with Aqua-Sim to perform the Packets Delivery Ratio (PDR) in \%, End-to-End Delay in seconds, Average Energy Consumptions in joules, Network Life Time in seconds, and Network Throughput in Kilobits per seconds (Kbps). The simulation parameters are taken in Table 1.

Table 1. Simulation parameters for NS2.30

\begin{tabular}{|l|l|}
\hline Parameters & Values \\
\hline Network Size & $1500 \mathrm{~m}$ x 1500m \\
\hline No. of Nodes & 300 \\
\hline Data Packet size & 64 byte \\
\hline Initial Energy & $70 \mathrm{~J}$ \\
\hline MAC Protocol (Shin \& Kim, 2008) & 802.11 -DYNAV \\
\hline Energy consumption for transmitting & $2 \mathrm{w}$ \\
\hline Energy consumption for receiving & $0.75 \mathrm{w}$ \\
\hline Energy consumption for idle listening & $8 \mathrm{mw}$ \\
\hline Transmission range & $100 \mathrm{~m}$ to $150 \mathrm{~m}$ \\
\hline Surface sink distance difference & $100 \mathrm{~m}$ \\
\hline Simulation time & $1000 \mathrm{sec}$ \\
\hline
\end{tabular}

\subsection{Packets Delivery Ratio (PDR)}

PDR is defined; the ratio (\%) delivered the data packets to sink nodes from source nodes. The PDR of CMSE2R is shown in Figure 5.

In Figure 5, the PDR of CME2R our proposed protocol is higher than CBE2R and EMGGR because CMSE2R utilizes the simple cluster formation with multipath mechanisms through use of minimum CDCM. On other hand; CBE2R and EMGGR are unable to maintain its PDR due to uncontrollable node mobility. 


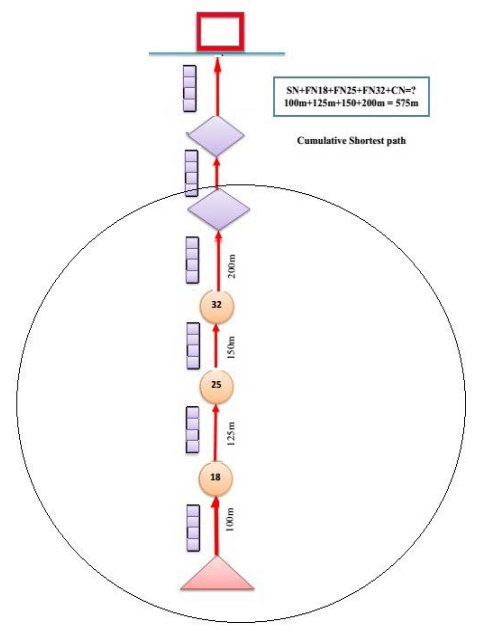

Figure 4. Packets forwarding from $\mathrm{SN}$ to $\mathrm{CN}$ through shortest CDCM.

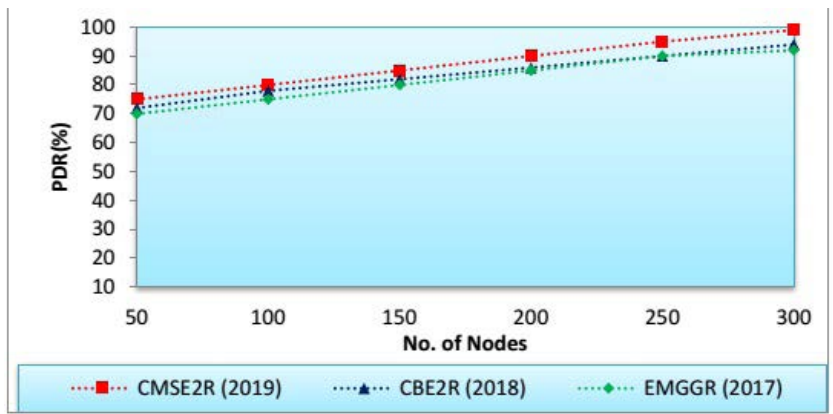

Figure 5. PDR of CMSE2R over CBE2R and EMGGR.

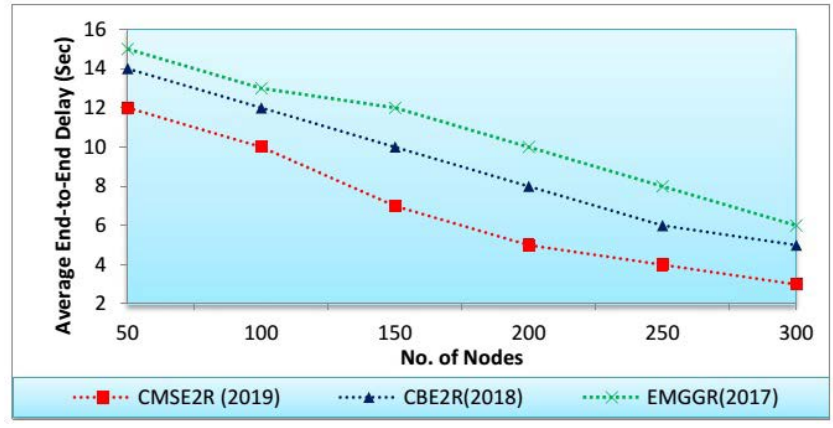

Figure 6. Average end-to-end delay of CMSE2R over CBE2R and EMGGR

\subsection{Average End-to-End Delay}

It can be measured that average delay of arrival of packets on sink nodes from all source nodes. The average end-toend delay is shown in Figure 6.

In Figure 6, the average end-to-end delay of CMSE2R is lower than CBE2R and EMGGR because the stable route and link quality between nodes reduces the end-toend delay. On other hand CBE2R and EMGGR are unable to maintain the link quality due to sparse uncontrollable network topology.

\subsection{Average Energy Consumption}

Average Energy Consumption can be calculated, the average difference of initial and final energy of the nodes. The average energy consumption of CMSE2R is shown in Figure 7.

In Figure 7, the average energy consumption of CMSE2R is lower than CBE2R and EMGGR because CMSE2R is based on CDCM. On other hand CBE2R and EMGGR cannot prolong their energy due to undefined energy mechanisms.

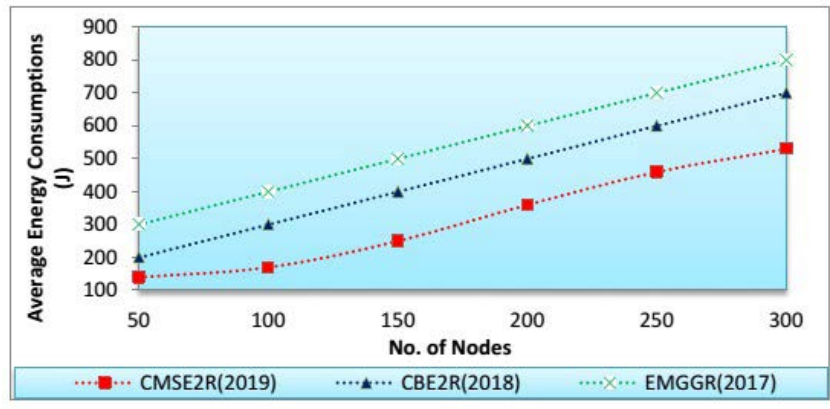

Figure 7. Average energy consumption of CMSE2R over CBE2R and EMGGR.

\subsection{Network Life Time}

Network Life Time can be defined on the die of any node in the entire network. The network lifetime of CMSE2R is shown in Figure 8.

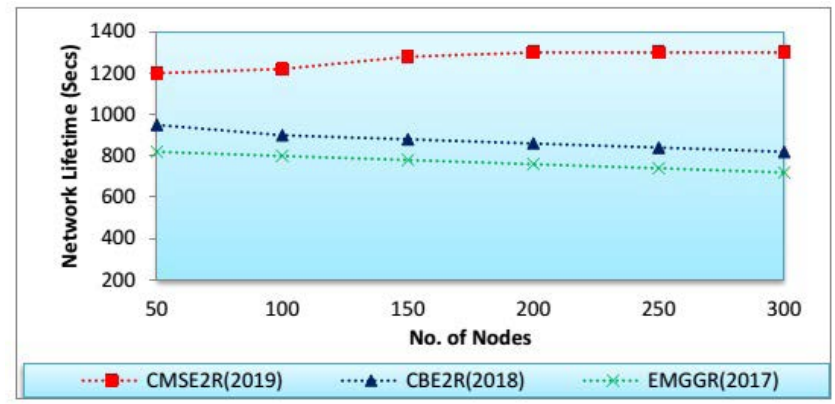

Figure 8. Network life time of CMSE2R over CBE2R and EMGGR.

The Network life time of CMSE2R is higher than CBE2R and EMGGR because CMSE2R utilizes the powerful static courier nodes with CDCM. On other hand the 
CBE2R and EMGGR cannot use such type of well manageable mechanisms.

\subsection{Network Throughput}

Network Throughput can be defined aggregate data rate achieved at the sink nodes. The network throughput of CMSE2R is shown in Figure 9.

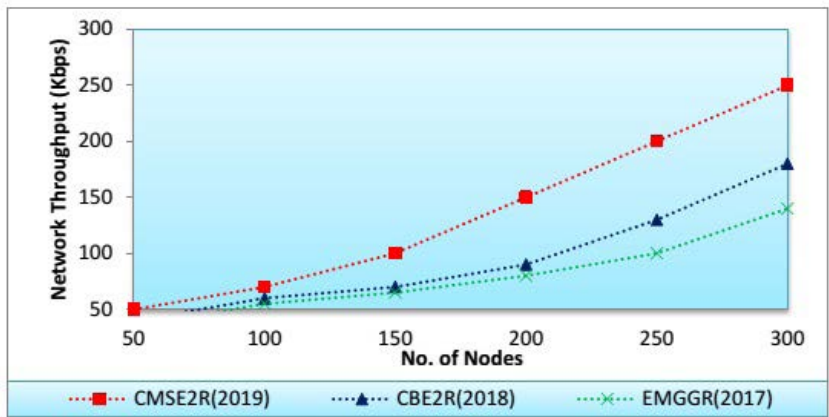

Figure 9. Network throughput of CMSE2R over CBE2R and EMGGR.

The network throughput of CMSE2R is higher than CBE2R and EMGGR because the shortest distance through $\mathrm{CDCM}$ is used. On other and the network throughput of CBE2R and EMGGR is lower than CMSE2R because when network become sparse then these both protocol are unable to maintain their network throughput.

\section{Conclusion}

In this research article, we proposed the Clustered-based Multipath Shortest-distance Energy Efficient Routing (CMSE2R) protocol for Underwater Wireless Sensor Network. CMSE2R is robust and well defined routing protocol which is based on four phases. In network setup phase we have deployed the Snods on water surface, we divided the water depth in upper and lower and deployed the fixed CNs in layer- 1 and layer- 2 in upper depth, where as in lower depth we deployed the FNs and SNs. From Layer-2, we made the static CNs as cluster-headnodes in cluster formation phase and make the clusters with FNs (cluster-member nodes) from CNs to SNs. In third phase in the relevant clusters, we made the multipath route development from source to courier nodes through CDCM with RREQ and RREP, in this phase the shortest route has been selected and data forwarding phase the data forwarding phase the packets are forwarded from shortest route from SNs to Snods. The results of CMSE2R are measured with CBE2R and EMGGR and the performance of CMSE2R shows the better results as compare to CBE2R and EMGGR,

\section{References}

1. Ahmed M. M2DFR: Multipath multi-layer data forwarding routing for underwater wireless sensor network. Indian Journal of Science and Technology. 2019; 12(1):1-6. https:// doi.org/10.17485/ijst/2019/v12i1/139794

2. Ahmed M. DCE2R: Distance Calculation Energy Efficient Routing Protocol for Underwater Wireless Sensor Network. IJCSNS International Journal of Computer Science and Network Security. 2018; 18(12):42-48.

3. Ahmed M, Salleh M, Channa MI. CBE2R: clustered-based energy efficient routing protocol for underwater wireless sensor network. International Journal of Electronics. 2018; 105(11):1916-30. https://doi.org/10.1080/00207217.2018.1 494323

4. Gul S, Jokhio SH, Jokhio IA. Light-weight depthbased routing for underwater wireless sensor network. International Conference Advancements in Computational Sciences (ICACS); 2018 https://doi.org/10.1109/ ICACS.2018.8333483

5. Gomathi R, Manickam JML. Energy efficient shortest path routing protocol for underwater acoustic wireless sensor network. Wireless Personal Communications. 2018; 98(1):843-56. https://doi.org/10.1007/s11277-017-4897-5

6. Ahmed M, Talpur F, Soomro MA. Comparative analysis on link-aware routing protocols for underwater wireless sensor network. International Journal of New Technologies in Science and Engineering. 2018; 5(5):1-7.

7. kyildiz IF, Pompili D, Melodia T. Underwater acoustic sensor networks: Research challenges. Ad hoc networks. 2005; 3(3):257-79. https://doi.org/10.1016/j.adhoc.2005.01.004

8. Gjanci P. Path finding for maximum value of information in multi-modal underwater wireless sensor networks. IEEE Transactions on Mobile Computing. 2018; 17(2):404-418. https://doi.org/10.1109/TMC.2017.2706689

9. Ahmed M, Salleh M, Channa MI. Routing protocols for underwater wireless sensor network based on location: A survey. Adhoc and Sensor Wireless Networks. 2017; 38:67101.

10. Ahmed M, Salleh M, Channa MI. Routing protocols based on node mobility for Underwater Wireless Sensor Network (UWSN): a survey. Journal of Network and Computer Applications. 2017; 78:242-52. https://doi.org/10.1016/j. jnca.2016.10.022

11. Ahmed M. Energy efficient routing protocols for UWSN: A Review. Telkomnika. 2017; 15(1). 
12. Kanthimathi N. Void handling using geo-opportunistic routing in underwater wireless sensor networks. Computers and Electrical Engineering; 2017. https://doi.org/10.1016/j. compeleceng.2017.07.016

13. Ahsan M. A recent study on routing protocols in UWSNs. International Journal of Advanced Computer Science and Applications. 2017; 8(4):431-5. https://doi.org/10.14569/ IJACSA.2017.080457

14. Faheem M, Tuna G, Gungor VC. LRP: Link quality-aware queue-based spectral clustering routing protocol for underwater acoustic sensor networks. International Journal of Communication Systems. 2017; 30(12):48-58. https://doi. org/10.1002/dac.3257

15. Ayaz M. An efficient dynamic addressing based routing protocol for underwater wireless sensor networks. Computer Communications. 2012; 35(4):475-86. https:// doi.org/10.1016/j.comcom.2011.11.014

16. Wan Z. An energy-efficient multi-level adaptive clustering routing algorithm for underwater wireless sensor networks.
Cluster Computing; 2018. p. 1-10. https://doi.org/10.1007/ s10586-018-2376-8

17. Wang Z. An energy-aware and void-avoidable routing protocol for underwater sensor networks. IEEE Access. 2018; 6:7792-801. https://doi.org/10.1109/ ACCESS.2018.2805804

18. Al Salti F, Alzeidi N, Arafeh BR. EMGGR: an energy-efficient multipath grid-based geographic routing protocol for underwater wireless sensor networks. Wireless Networks. 2017; 23(4):1301-14. https://doi.org/10.1007/s11276-016-1224-0

19. Chao CM, Jiang CH, Li WC. DRP: An energy-efficient routing protocol for underwater sensor networks. International Journal of Communication Systems. 2017; 1:1-24. https:// doi.org/10.1002/dac.3303

20. Rahman Z. Reliable and Energy Efficient routing Protocol (REEP) for Underwater Wireless Sensor Networks (UWSNs). IEEE 12th Malaysia International Conference on Communications (MICC); Kuching, Sarawak, Malaysia. 2015. p. 24-9. 